\title{
Hydrogen on Graphene Under Stress: Molecular Dissociation and Gap Opening
}

\author{
Hayley McKay, ${ }^{1}$ David J. Wales, ${ }^{1}$ S.J. Jenkins, ${ }^{1}$ J.A. Verges,${ }^{2}$ and P.L. de Andres ${ }^{2}$ \\ 1 University Chemical Laboratories, \\ Lensfield Road, Cambridge CB2 1EW, UK \\ 2 Instituto de Ciencia de Materiales de Madrid \\ (CSIC) E-28049 Cantoblanco, Madrid, SPAIN
}

(Dated: October 22, 2018)

\begin{abstract}
Density functional calculations are employed to study the molecular dissociation of hydrogen on graphene, the diffusion of chemisorbed atomic species, and the electronic properties of the resulting hydrogen on graphene system. Our results show that applying stress to the graphene substrate can lower the barrier to dissociation of molecular hydrogen by a factor of six, and change the process from endothermic to exothermic. These values for the barrier and the heat of reaction, unlike the zero stress values, are compatible with the time scales observed in experiments. Diffusion, on the other hand, is not greatly modified by stress. We analyse the electronic structure for configurations relevant to molecular dissociation and adsorption of atomic hydrogen on a graphene single layer. An absolute band gap of $0.5 \mathrm{eV}$ is found for the equilibrium optimum configuration for a narrow range of coverages $(\theta \approx 0.25)$. This value is in good agreement with experiment [Elias et al., Science 323, 610 (2009)].

PACS numbers: 68.43.-h,68.43.Bc,81.05.Uw

Keywords: carbon, graphene, hydrogen, molecular hydrogen dissociation, reaction mechanisms, stress engineering, density functional theory
\end{abstract}




\section{INTRODUCTION}

The safe and efficient storage of hydrogen is a crucial step towards its use in the future as an energy vector. The U.S. Department of Energy (DOE) has launched an important challenge by funding research to overcome the threshold of $6.5 \mathrm{~kg}$ of stored hydrogen per $100 \mathrm{~kg}$ of total storage system weight. 1 In the present contribution we focus on the 'gas adsorbed on solid' approach, with graphene layers as the supporting material. Adsorption of hydrogen on graphene $(\mathrm{G})$ can take place in different ways: (i) physisorption of molecular hydrogen, (ii) physisorption of atomic hydrogen, (iii) chemisorption of atomic hydrogen, (iv) chemisorption of small clusters of atomic hydrogen. These scenarios exhibit different adsorption/desorption energies, optimum geometrical configurations and diffusion barriers. A good understanding of the interplay between molecular physisorption and atomic chemisorption would be useful for addressing the problem of hydrogen storage, as well as for understanding fundamental issues, such as the puzzle of how $\mathrm{H}_{2}$ is formed in the interstellar medium. ${ }^{2}$

Graphene itself is currently of particular interest due to the recently developed capability for obtaining samples consisting of single or a few layers. $\stackrel{3}{3}$ Such layers provide an excellent model for advancing the applications of related materials, such as graphite and nanotubes. In particular, graphite is an attractive candidate for hydrogen storage because it is cheap, chemically inert and environmental friendly. However, theoretical and experimental studies have cast doubts about achieving the goal proposed by the DOE, with certain exceptions that have focused in the quantum properties of such a light element as hydrogen.1

To investigate the feasibility of loading and releasing hydrogen efficiently in graphite or carbonaceous minerals we have characterised diffusion and dissociation barriers for hydrogen on graphene under stress. External and internal stresses have been reported to induce

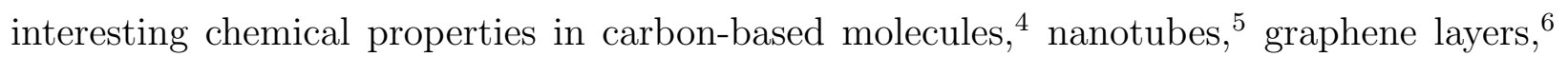
etc. We find that on applying a moderate amount of stress the equilibrium state and the barriers for molecular dissociation become much more favourable.

Understanding the hydrogenation of graphene layers is important from several perspectives. Recently, in a series of elegant experiments, Elias et al. ${ }^{7}$ have demonstrated the reversible transition of graphene layers from a semimetallic to a semiconducting regime upon adsorption of hydrogen. This is an interesting observation, which should help to advance the application of graphene layers in microelectronics. Our results should provide insight 
into these experiments, particularly the conditions required to create an absolute band gap in the graphene semi-metallic band structure, in terms of the adsorbate coverage.

\section{A. Methods}

Our calculations are based on density functional theory (DFT) ${ }^{8}$ We calculated the adsorption energies, dissociation pathways, and barriers for atomic and molecular hydrogen on a $3 \times 3$ periodic supercell with a vacuum gap in the perpendicular direction of 10 to 20 $\AA$. The wavefunctions were expanded in a plane-wave basis set up to a cutoff of $350 \mathrm{eV}$ and were sampled on a Monkhorst-Pack $6 \times 6 \times 1$ mesh inside the Brillouin zone. Electronic bands were obtained using a smearing width of $\eta=0.01 \mathrm{eV}$. Carbon and hydrogen atoms were described by soft pseudopotentials. ${ }^{9}$ The choice of the exchange and correlation (XC) potential is an important aspect of DFT calculations. We considered both the local density approximation (LDA $)^{10}$ and a generalized gradient approximation (GGA) functional, RPBE, 11 to check the influence of that choice on our results. This formalism is accurate enough to reproduce structural and electronic properties of chemisorbed species, but cannot accurately describe the physisorption regime, because the non-local correlation effects needed to properly describe weak van der Waals interactions are not included. The energy differences of interest here, and their variation with external stress, are relatively insensitive to whether the LDA or RPBE functional is used. Furthermore, the errors associated with the DFT formalism at large distances are unimportant, because the energies involved in making/breaking chemical bonds are much greater than the few meV associated with van der Waals interactions.

Total energies and gradients were computed with the CASTEP program, ${ }^{12}$ interfaced to OPTIM $^{13}$ for geometry optimisation. All the transition states reported below were refined using the gradient-only version of hybrid eigenvector-following 14 with a convergence condition of $0.01 \mathrm{eV} / \AA$ for the root mean square gradient. Approximate steepest-descent paths were calculated by energy minimisation for each transition state to characterise the corresponding pathway using the limited-memory Broyden-Fletcher-Goldfarb-Shanno (LBFGS) algorithm,,$\frac{17}{17}$ following displacements of order $0.01 \AA$ parallel and antiparallel to the Hessian eigenvector corresponding to the unique negative Hessian eigenvalue. 


\section{RESULTS AND DISCUSSION}

The most favourable configuration for hydrogen on graphene corresponds to molecular

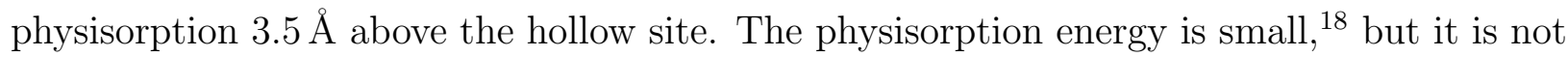
relevant here, since we are dealing with processes involving much larger changes in energy, such as the formation of a hydrogen-carbon bond, or breaking the bond in the hydrogen molecule. In contrast, for atomic hydrogen the most favourable configuration involves chemisorption directly on top of a carbon atom. RPBE calculations show that the C-H bond

length is then $1.1 \AA$, corresponding to an adsorption energy of $E_{G+H}-E_{G}-\frac{1}{2} E_{H_{2}}=+1.68 \mathrm{eV}$, while the carbon directly below is puckered upwards by $0.4 \AA$. It is important to notice that chemisorption of atomic hydrogen interferes with the strong $\mathrm{sp}^{2}$ sigma bonds of planar graphene, producing an energetic cost derived from the elastic deformation of the substrate. Hydrogen atoms approaching a graphene surface from the gas phase therefore encounter a barrier in reaching the chemisorbed state. This barrier depends on the position on the surface, varying between $0.28 \mathrm{eV}$ and $1.27 \mathrm{eV}$ for atop and hollow sites (RPBE functional).

Both atomic and molecular hydrogen are trapped on the graphene surface in a relatively shallow physisorption well at large distances $(\geq 3.5 \AA$ for the RPBE functional). The diffusion barriers for physisorption are low, and hence the adsorbed species are mobile. However, physisorbed species are subject to a correspondingly high desorption probability; at room temperature (RT) physisorbed atomic or molecular hydrogen desorb on a time scale of nanoseconds. The only way to keep such a system stable on a reasonable time scale for practical applications is to reach a chemisorbed state. While hydrogen molecules do not chemisorb, atomic hydrogen can form strong bonds on atop sites, provided that (i) the molecule initially dissociates and (ii) atoms can find the favourable region where barriers between physisorbed and chemisorbed minima are small. The values we obtain for the barriers imply that at RT a hydrogen atom would find its way to the chemisorbed well on a time scale of nanoseconds near the atop region, while it would take years for chemisorption to occur via a trajectory only involving the local minimum corresponding to the hollow site. To meet the DOE's challenge we need to consider the formation and dissociation of the hydrogen molecule in the high coverage regime. The reaction $\mathrm{H}_{2} \rightarrow 2 \mathrm{H}$ near a free-standing graphene sheet is associated with a barrier of $3.29 \mathrm{eV}$ and an endothermic heat of reaction of $1.89 \mathrm{eV}$, making dissociation of molecular hydrogen a rare event (RPBE values quoted 
here). Furthermore, to load a stack of graphene layers efficiently we need to consider the diffusion barriers for atomic hydrogen. Our calculations show that chemisorbed hydrogen diffuses from top site to top site via bridge sites with an associated barrier of $0.98 \mathrm{eV}$. External stress increases the diffusion barrier by $9 \%$ and $60 \%$ for tensile and compressive strains of $\epsilon=0.1$ and -0.05 , respectively (Figure 1). These barriers result in diffusion times that are too long for effective loading of chemisorbed hydrogen in graphite at RT $\left(\approx\left(10^{4} \mathrm{~s}\right.\right.$ for a single hop), leaving diffusion of molecular hydrogen or physisorbed atomic species as the most practical scenarios. Diffusion through the hollow site is unfavourable, involving a barrier of $4.05 \mathrm{eV}$, resulting in a practically impenetrable layer in the perpendicular direction at RT, in good agreement with experiment.7

\section{A. $\mathbf{H}_{2}$ Dissociation on Graphene}

We now examine the effect of internal and external stress on $\mathrm{H}_{2}$ dissociation/formation. Figure 2 shows how the barriers and reaction energies for the dissociation process are affected by external stress. The deformations considered here are quite realistic for graphene, a material that can accommodate tensile strains up to $\epsilon=0.25$ (corresponding to a stress of $\sigma \approx 42 \mathrm{Nm}^{-1}$ ) before breaking up $\frac{19}{19}$ Compressive strains of up to $\epsilon=-0.05$ have also been measured from diffraction experiments in hydrogenated samples ${ }^{7}$ (our calculations corroborate that these samples tend to buckle on an atomic scale, at least in the region around the adsorbates). Indeed, $\mathrm{C}-\mathrm{C}$ bonds can accommodate a wide range of values, from $1.37 \AA$ for triple bonds (sp hybridisation) to $1.54 \AA$ for single bonds ( $\mathrm{sp}^{3}$ hybridisation), with a typical minimum value in allenes of $1.30 \AA$ and a maximum observed bond length of $1.78 \AA .4$ For tensile stresses related to uniform deformations in the 2D lattice between $\epsilon=0.10$ and 0.15 the barriers are not strongly affected, but the heat of reaction for molecular hydrogen dissociation changes from endothermic $(\Delta E=1.89 \mathrm{eV}$ for $\epsilon=0)$ to exothermic $(\Delta E=$ $-0.88 \mathrm{eV}$ at $\epsilon=0.15$ ). These results (values quoted for RPBE) show that between RT and $500 \mathrm{~K}$, physisorbed molecular hydrogen would not transform to chemisorbed atomic hydrogen on a reasonable time scale. In contrast, a compressive strain introduces a totally different scenario: for $\epsilon=-0.05$ the RPBE barrier drops to $2.19 \mathrm{eV}$, improving the predicted time for dissociation by about a factor of $10^{11}$; for $\epsilon=-0.1$ the barrier decreases to $0.59 \mathrm{eV}$, which should allow a single physisorbed $\mathrm{H}_{2}$ molecule to dissociate on a time scale of milliseconds at 
RT. These RPBE values are expected to compare well with experiment, since good accuracy has been obtained for similar systems, with a slight tendency to overestimate the barriers.11 To assess the influence of the XC model we compare with LDA, to obtain lower bounds for these barriers. The LDA barrier for $\epsilon=0(2.38 \mathrm{eV})$ drops to $1.4 \mathrm{eV}$ under a compressive strain of $\epsilon=-0.05$, and to $0.04 \mathrm{eV}$ under a compressive strain of $\epsilon=-0.1$. The rate of change of the barrier with respect to external stress is therefore similar for both functionals (changes of $2.70 \mathrm{eV}$ and $2.34 \mathrm{eV}$ for $\Delta \epsilon=-0.1$ ), highlighting the consistency of predicted trends derived from introducing stresses to the system. Different XC models produce slightly different values for the strain threshold necessary for dissociation to occur on a given time scale, but our best estimate of $\epsilon=-0.05$ to -0.1 is narrow enough to be significant. Furthermore, we remark that the reaction would quickly reach equilibrium, due to the more favourable exothermic heat of reaction for compression (Figure 2, lower curve).

We therefore predict that the pathway for molecular dissociation near stressed graphene enables the reaction to occur much faster. Elias et al. have recently demonstrated that a single graphene layer can be hydrogenated to open a band gap of about $0.5 \mathrm{eV} !^{7}$ Transmission electron microscopy provides evidence for a non-uniform distribution of stresses, resulting in measurable variations of the $2 \mathrm{D}$ unit cell parameter ranging from $-0.05 \%$ (compressive) to $0.03 \%$ (tensile). Hydrogenation is performed by exposing graphene to a low pressure hydrogen-argon mixture $\left(10 \% \mathrm{H}_{2}\right)$ at about $600 \mathrm{~K}$. This process is reversible, and mass spectrometry has been used to detect molecular hydrogen leaving the substrate after annealing to about the same temperature. From our results we interpret the experimental evidence in the following way. Starting with physisorbed molecular hydrogen, a few molecules dissociate to produce chemisorbed atomic hydrogen on a time scale of $\tau \approx 1 /\left(10^{16} \times 10^{12} \times e^{\left(-B / k_{B} T\right)}\right)$, where $10^{12} \mathrm{~Hz}$ represents a typical attempt frequency, $10^{16}$ is the number of molecules in contact with the surface per mole of hydrogen, and the Boltzmann factor defines the dependence on the barrier, $B$, for an effective surface temperature, $T$. At about $300^{\circ} \mathrm{C}$ a few molecules should start to dissociate on a time scale of seconds, and the chemisorbed hydrogen induces buckling on the surface, creating a local region where the substrate is under an effective compressive stress. According to our results, this stress lowers the barrier for dissociation (Figure 2) and helps to dissociate other molecules, the chemisorbed atomic hydrogen cannot diffuse away at a noticeable rate and a cluster of atomic hydrogen is formed around that region. Atomic hydrogen is therefore preferentially adsorbed in strained regions. Strains 
and stresses derived from the adsorption of hydrogen are well inside the elastic regime, and graphene layers can recover their original geometry after annealing to $300^{\circ} \mathrm{C}$, when atomic hydrogen desorbing from the surface recombines to form molecular hydrogen, which is the species detected. This picture is supported by STM experiments, where clustering of hydrogen atoms due to preferential sticking has been observed.20 In this interpretation, the mechanism responsible for the preferential sticking is the modification of $\mathrm{C}-\mathrm{C}$ bonds under local stress induced by neighbouring chemisorption of hydrogen. The scenario proposed here is physically reasonable and backed up by state-of-the-art calculations that indicate how dissociation can occur on a time scale of days rather than years.

It has been suggested that clustering is related to the elastic energy penalty paid for clustered hydrogen vs. maximally dispersed hydrogen at sub-monolayer coverages. A careful consideration of this point is beyond the scope of this work, but nevertheless we can estimate the effect of clustering on the elastic deformation of the substrate by comparing total energies of the carbon atoms alone frozen in their equilibrium positions due to the presence of hydrogen. In particular, in a $2 \times 2$ supercell, chemisorbing a single hydrogen produces an elastic deformation in the substrate that requires $0.88 \mathrm{eV}$. The corresponding deformation for two hydrogen atoms located in their optimum position (nnNN site) requires $2.09 \mathrm{eV}$. Therefore, positioning a second hydrogen in the vicinity of the first one carries a significant energetic penalty, and suggests to us that clustering is better explained in terms or our proposed mechanism than by a balance of elastic deformations. However, further work will be required to resolve this question.

\section{B. Electronic Structure Effects}

After molecular dissociation and chemisorption of the individual hydrogen atoms the characteristic graphene semi-metallic electronic band structure is perturbed and partial gaps open in some directions in the Brillouin zone. However, for the system to acquire semiconducting properties, an absolute gap must appear in the density of states (DOS). We find that such an absolute gap is sensitive to the coverage, which allows us to provide an estimate for comparison with experiment. The origin of this gap is related to the periodic potential created by the accumulation of charge around the $\mathrm{C}-\mathrm{H}$ bond and/or by the electronic band shifts originating in the local modifications of the $\mathrm{C}-\mathrm{H}$ bonding orbital. ${ }^{21}$ Figures 3 and 4 
show the band structure and density of states corresponding to the different configurations we have considered for two chemisorbed hydrogen atoms in a $3 \times 3$ cell $(\theta=1 / 9)$. Here we describe the LDA results, but the GGA calculations are essentially the same.

For clean graphene near the $\mathbf{K}$ point we observe the distinctive linear crossing of bands at the Fermi energy responsible for semi-metal character. Physisorbed atomic and molecular hydrogen do not significantly change this behaviour. The next interesting feature is the appearance of a half-filled non-dispersing band at the Fermi level upon chemisorption of a single hydrogen atom. ${ }^{21}$ Adsorption of a second hydrogen atom after molecular dissociation can occur in three neighbouring sites: (i) nearest-neighbour position (NN), (ii) next-toNN position (nNN), or (iii) next-to-next-NN (nnNN). According to Lieb's theorem, for a bipartite lattice like graphene the ground state for $\mathrm{NN}, \mathrm{nNN}$ and $\mathrm{nnNN}$ configurations is attained with $S=0,2$, and $0 \mu_{B}$, respectively. ${ }^{22}$ Although Lieb's theorem is only strictly valid for the Hubbard Hamiltonian, we have found that it correctly predicts the optimum spin configurations obtained using DFT. The global minimum is related to occupancy of nnNN sites, while the next most stable is the NN position. The intermediate site (nNN) is the least stable one; it is a magnetic solution, and spontaneously evolves to nnNN under the restriction of $S=0$. From an electronic point of view, bands associated with the nNN site appear similar to the ones for only one hydrogen atom, showing that direct interaction between atoms at this distance is weak. However, interaction between adsorbates mediated by the substrate through static elastic distortions can be important and should not be neglected. These distortions produce a departure from a flat geometry and are favourable, so long as they promote the $\mathrm{sp}^{2}$ to $\mathrm{sp}^{3}$ transformation. For high coverages, chemisorption on nnNN sites opens an absolute gap allowing the system to find a more stable configuration. The absolute band gap evolves from $3.1 \mathrm{eV}$ for $\theta=1 / 4$ to $0.6 \mathrm{eV}$ for $\theta=2 / 9$. Below and above these two values for the hydrogen coverage the computed band gap is too small or too large compared with experiment. Therefore, we conclude that the actual coverage in ref. 7 should be near $\theta \approx 1 / 4$, and the geometrical configuration near to the optimum equilibrium one. It is interesting to notice that occupation of the alternative locations ( $\mathrm{NN}$ and $\mathrm{nNN})$ is not only a non-optimal configuration, but more crucially it does not result in an absolute gap in the density of states even for the larger coverages considered $(\theta=1 / 4)$. A characteristic double peak appears (Figure 4), similar to that found for chemisorption of a single hydrogen atom, where only a direct gap at the $\mathbf{K}$ point appears (not an absolute one) ${ }^{21}$ These results 
can be understood in terms of topological connectivity: if we assume that the $\mathrm{C}-\mathrm{H}$ bond saturates the $\mathrm{p}_{z}$ electron on that site, occupation of nnNN sites for $\theta \geq 2 / 9$ breaks the graphene layer in a set of nearly disconnected hexagons where first-neighbour interactions are too weak and result in a series of narrow peaks and an absolute gap (inset and shaded curve in Figure 4). For $\theta=1 / 4$ the rings become totally disconnected and a larger gap appears. This kind of reconstruction is reminiscent of a Peierls distortion, where the system minimises its energy by a rehybridization of the basis in the unit cell under a new balance between kinetic and potential energy contributions. While the popular $2 \times 2$ tight-binding Hamiltonian including only $\pi$ electrons reproduces most of the low energy physics correctly, a proper account of the above effect requires consideration of the in-plane $\mathrm{sp}^{2}$ sigma bonds responsible for the elastic contribution to the energy.

\section{Conclusions}

In summary, we have studied the effect of external stress on diffusion of atomic chemisorbed hydrogen and on the dissociation of the $\mathrm{H}_{2}$ molecule. While external stress has little influence on diffusion barriers we find that compression changes the endothermic dissociation of physisorbed $\mathrm{H}_{2}$ to exothermic, and lowers the barrier by about $2.5 \mathrm{eV}$ $(\epsilon=-0.1)$ for both the LDA and GGA functionals considered. Dissociation of $\mathrm{H}_{2}$ is the first step for activated chemisorption of hydrogen atoms, and leads to a significant strain in the substrate. This strain lowers the barrier for further chemisorption, favouring the pairing of chemisorbed species over a small region related to the elastic distortion created by the formation of the first C-H bond. The most favourable configuration for a pair of chemisorbed hydrogens is a paramagnetic ground state occupying the next-to-next-nearest-neighbour site, while the second most favourable is the nearest-neighbour site. The intermediate site becomes less favourable in accord with Lieb's theorem, which predicts a ferromagnetic solution of spin $2 \mu_{B}$. The electronic structure of these paired chemisorbed hydrogen atoms is such that the graphene-covered layer becomes semiconducting for occupancy of nnNN sites at coverages near $\theta=2 / 9$ or greater, while it remains semimetallic for $\mathrm{NN}$ and $\mathrm{nNN}$ sites, even for coverages up to $\theta=1 / 4$. These results should help in the rational design of hydrogen storage systems based upon carbon substrates: a graphene layer exposed to molecular

hydrogen would form a physisorbed species with low barriers to diffusion. External com- 
pressive stress in the range between $5 \%$ to $10 \%$ helps to dissociate molecules to form atomic chemisorbed hydrogen. These are bound to graphene with a low dissociation probability, and diffuse slowly on the surface. Releasing the external stress would induce the reverse reaction, favouring the formation of molecular hydrogen, with a low desorption barrier.

Financial support from the Spanish CYCIT is acknowledged (MAT2008-1497, CSD200741 NANOSELECT, and FIS2009-8744).

1 S. Patchkovskii, J. Tse, S. Yurchenko, L. Zhechkov, T. Heine, and G. Seifert, Proc. Natl. Acad. Sci. U.S.A. 102, 10439 (2005), doi:10.1073/pnas.0501030102.

2 L. Hornekaer, Z. Sljivancanin, W. Xu, R. Otero, E. Rauls, I. Stensgaard, E. Laegsgaard, B. Hammer, and F. Besenbacher, Phys. Rev. lett. 96, 156104 (2006), doi:10.1103/PhysRevLett.96.156104.

3 K. S. Novoselov, Science 306, 666 (2004), doi:10.1126/science.1102896.

4 R. Hoffmann and H. Hopf, Angew. Chem. Int. Ed. 47, 4474 (2008), doi:10.1002/anie.200705775.

5 D. Srivastava, D. W. Brenner, J. D. Schall, K. D. Ausman, M. Yu, and R. S. Ruoff, J. Phys. Chem. B 103, 4330 (1999), doi:10.1021/jp990882s.

6 P. L. de Andres and J. A. Verges, Appl. Phys. Lett. 93, 171915 (2008), doi:10.1063/1.3010740.

7 D. C. Elias, R. R. Nair, T. M. G. Mohiuddin, S. V. Morozov, P. Blake, M. P. Halsall, A. C. Ferrari, D. W. Boukhvalov, M. I. Katsnelson, A. K. Geim, et al., Science 323, 610 (2009), doi:10.1126/science.1167130.

8 P. Hohenberg and W. Kohn, Phys. Rev. 136, B864 (1964), doi:10.1103/PhysRev.136B864.

9 D. Vanderbilt, Phys. Rev. B 41, 7892 (1990), doi:10.1103/PhysRevB.41.7892.

10 W. Kohn and L. J. Sham, Phys. Rev. 140, A1133 (1964), doi:10.1103/PhysRev.140.A1133.

11 B. Hammer, L. B. Hansen, and J. K. Norskov, Phys. Rev. B 59, 7413 (1999), doi:10.1103/PhysRevB.59.7413.

12 S. J. Clark, M. D. Segall, C. J. Pickard, P. J. Hasnip, M. J. Probert, K. Refson, and M. C. Payne, Z. fuer Kristallographie 220, 567 (2005), doi:10.1524/zkri.220.5.567.65075.

13 (OPTIM); http://www-wales.ch.cam.ac.uk/software.html.

14 L. J. Munro and D. J. Wales, Phys. Rev. B 59, 3969 (1999), doi:10.1103/PhysRevB.59.3969.

15 Y. Kumeda, L. J. Munro, and D. J. Wales, Chem. Phys. Lett. 341, 185 (2001), 
doi:10.1016/S0009-2614(01)0034-7.

16 D. J. Wales, Energy Landscapes (Cambridge Univ. Press, 2003).

17 D. Liu and J. Nocedal, Math. Prog. 45, 503 (1989), doi:10.1007/BF01589116.

18 M. Bonfanti, R. Martinazzo, G. Tantardini, and A. Ponti, J. of Phys. Chem. C 111, 5825 (2007), doi:10.1021/jp070616b.

19 C. Lee, X. Wei, J. Kysar, and J. Hone, Science 321, 385 (2008), doi:10.1126/science.1157996.

20 L. Hornekaer, E. Rauls, W. Xu, Z. Sljivancanin, R. Otero, I. Stensgaard, E. Laegsgaard, B. Hammer, and F. Besenbacher, Phys. Rev. Lett. 97, 186102 (2006), doi:10.1103/PhysRevLett.97.186102.

21 E. J. Duplock, M. Scheffler, and P. J. D. Lindan, Phys. Rev. Lett. 92, 225502 (2004), doi:10.1103/PhysRevLett.92.225502.

22 E. H. Lieb, Phys. Rev. Lett. 62, 1201 (1989), doi:10.1103/PhysRevLett.62.1201. 


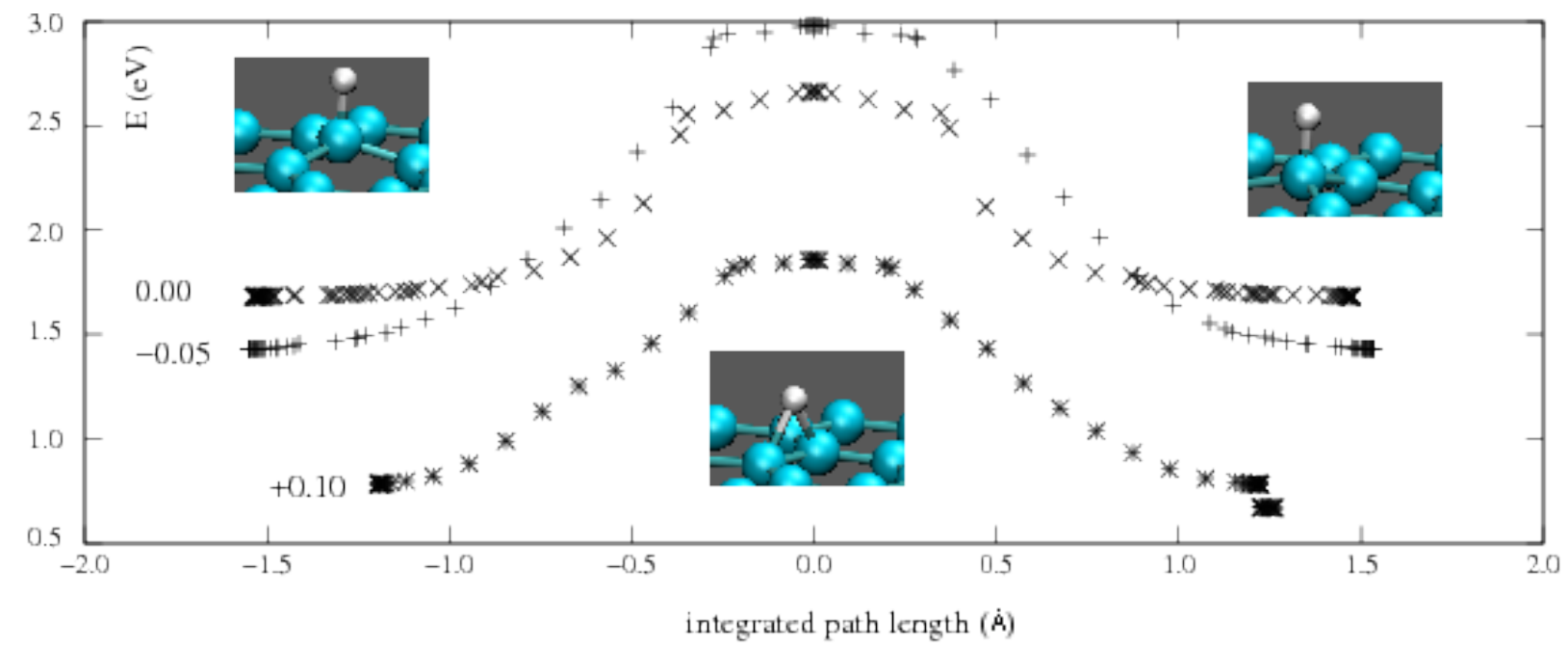

FIG. 1: Energy/eV (RPBE functional11 ${ }^{11}$ ) as a function of integrated path length (in $\AA$ ) for diffusion of chemisorbed atomic hydrogen on graphene under different uniform stresses characterised by the corresponding unit cell strains $(\epsilon=+0.1$, tensile; $\epsilon=-0.05$, compressive). The structures of the transition state and minima are superimposed.

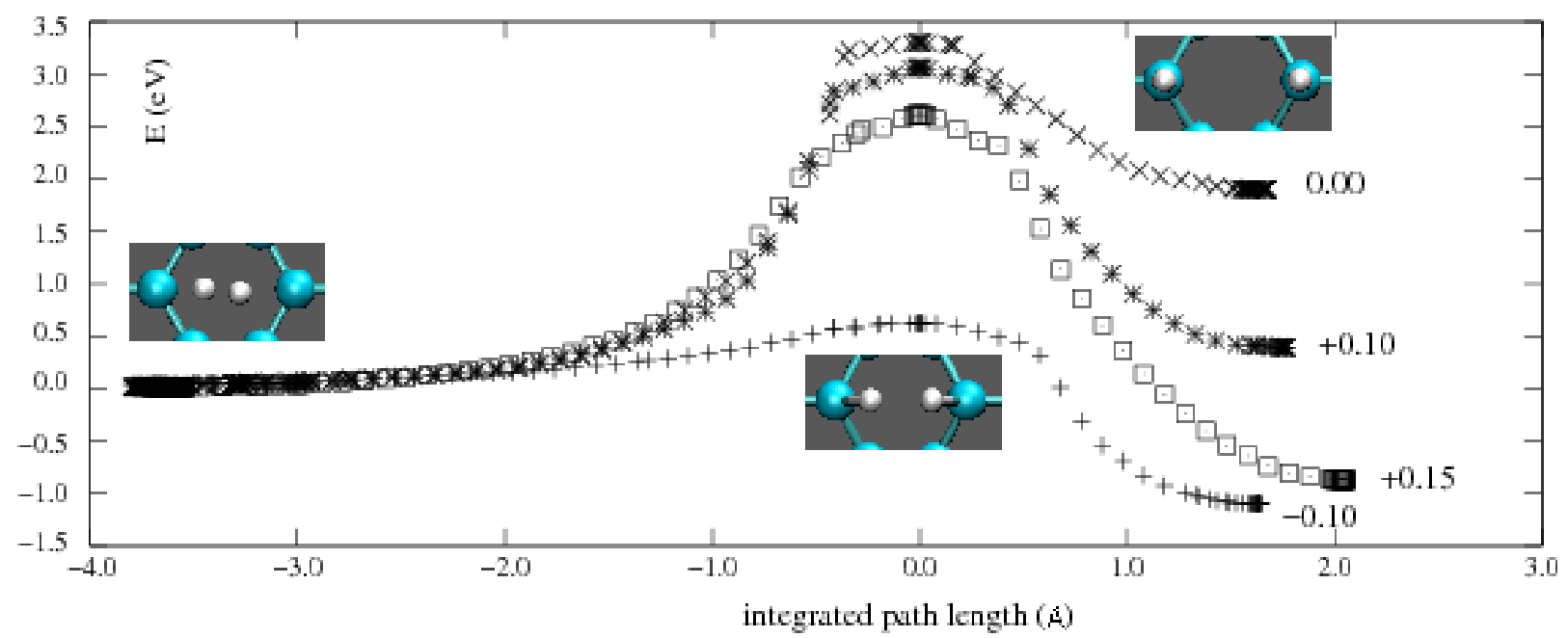

FIG. 2: Energy profile/eV (RPBE functional11) as a function of integrated path length (in $\AA$ ) for $\mathrm{H}_{2} \rightarrow 2 \mathrm{H}$ under different compressive/tensile strains, $\epsilon$. The structures of the transition state and minima are superimposed. 


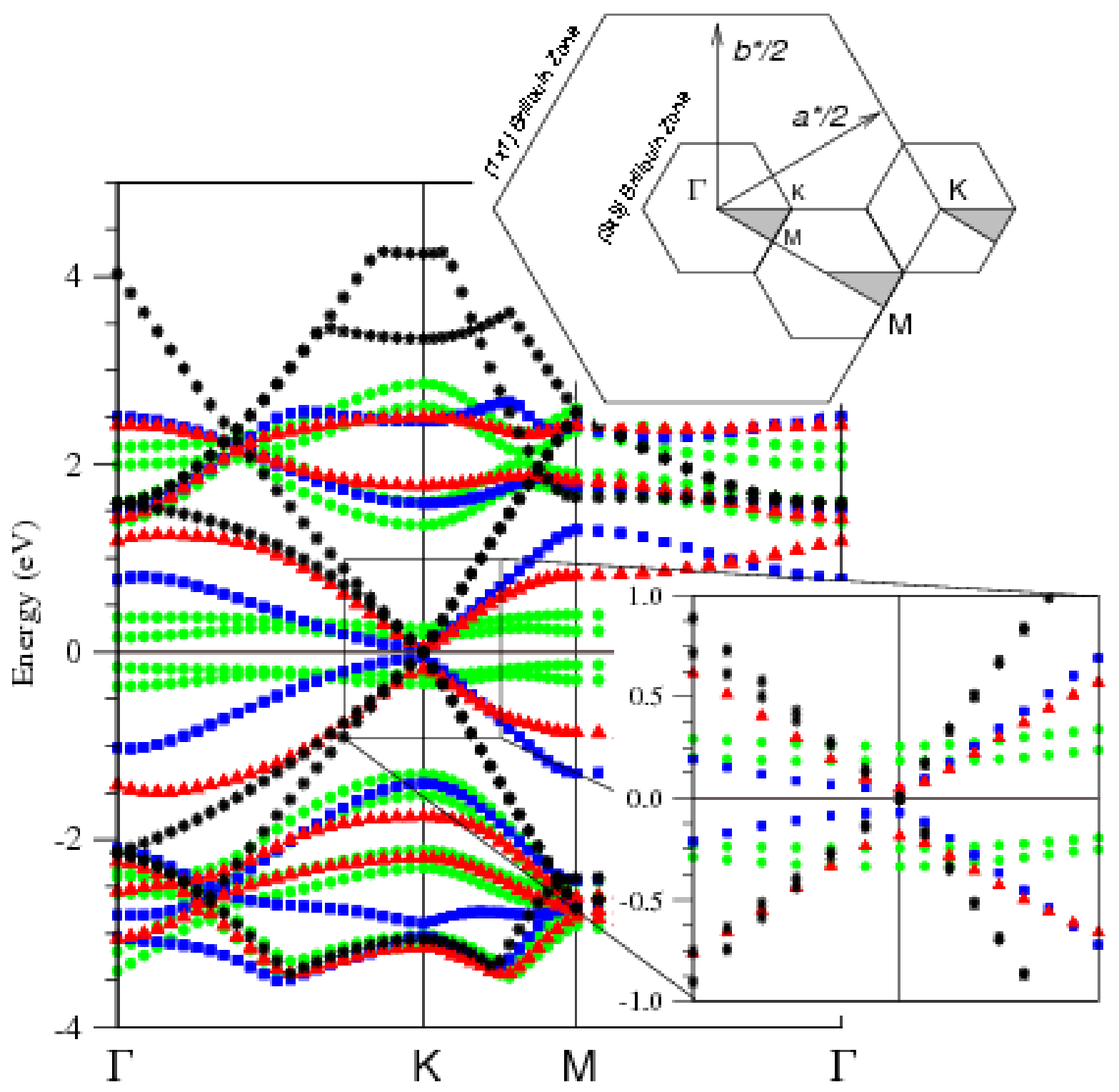

FIG. 3: Electronic band structure for clean graphene and hydrogen adsorbed on graphene $(\theta=1 / 9)$ : Clean graphene (circles, black), $\mathrm{H}+\mathrm{H}(\mathrm{nnNN}) /$ graphene (triangles-up, red), $\mathrm{H}+\mathrm{H}(\mathrm{nNN})$ /graphene (squares, green), $\mathrm{H}+\mathrm{H}(\mathrm{NN}) /$ graphene (triangles-down, blue). The central zone around $\mathbf{K}$ has been magnified on the right. Inset: reciprocal-space path $(\Gamma-\mathbf{K}-\mathbf{M}-\Gamma)$ in the $3 \times 3$ irreducible Brillouin zone and its relationship to the $1 \times 1$ zone. 


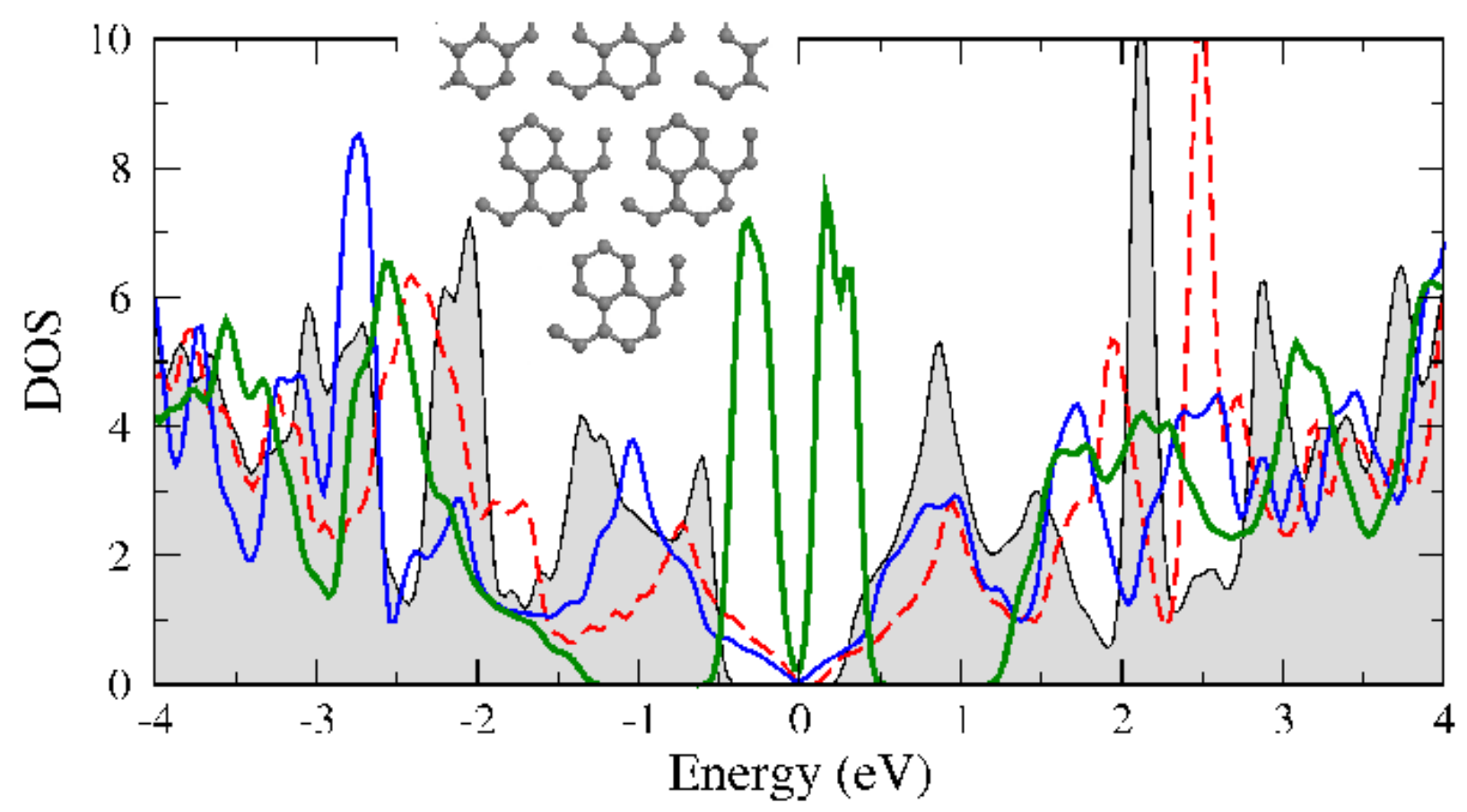

FIG. 4: Density of states $\left(\mathrm{eV}^{-1}\right.$, smearing width $\left.\eta=0.05 \mathrm{eV}\right)$ for the following configurations (coverage, $\theta=1 / 9$ ): nnNN (red, short-dashed), $\mathrm{nNN}$ (green, dotted) and $\mathrm{NN}$ (blue, dot-dashed). The shaded curve corresponds to $\mathrm{nnNN}$ at $\theta=2 / 9$ where an absolute gap of $0.63 \mathrm{eV}$ appears, and the inset illustrates the connectivity for this case. The Fermi energy is aligned at the origin. 